\title{
A Case Report of an Ultrasound-Guided and Fluoroscopy-Assisted Posterior Approach for Celiac Plexus Neurolysis in a Patient with Pancreatic Cancer-Associated Abdominal Pain
}

This article was published in the following Dove Press journal:

Journal of Pain Research

\section{Rong Shi \\ Danxu Ma \\ Yinan Yang \\ Yun Wang (DD}

Department of Anesthesia and Pain Medicine, Beijing Chaoyang Hospital, Capital Medical University, Beijing 100020, People's Republic of China
Correspondence: Yun Wang Department of Anesthesia and Pain Medicine, Beijing Chaoyang Hospital, Capital Medical University, No. 8, Gongtinan Road, Chaoyang District, Beijing 100020, People's Republic of China Email wangyun129@ccmu.edu.cn
Background: Imaging-guided celiac plexus neurolysis using ultrasound (US) guidance via a transabdominal approach and endoscopic-ultrasound (EUS) has been increasingly applied for the treatment of pancreatic cancer-associated abdominal pain.

Objective: To investigate the application of ultrasound-guided and fluoroscopy-assisted celiac plexus neurolysis in a patient with advanced pancreatic cancer suffering from refractory abdominal pain for which oral opioid treatment was ineffective.

Case Report: We report a case of ultrasound-guided and fluoroscopy-assisted celiac plexus neurolysis in a patient with advanced pancreatic cancer with refractory abdominal pain. With the patient in the prone position, celiac plexus neurolysis was performed under real-time US guidance. The transducer was placed below the costal margin and a puncture needle with an ultrasound enhancement tip was inserted in-plane aiming for the lateral anterior end of the vertebral body. The correct needle tip position was confirmed by the C-arm with contrast material located anterior to the vertebral body and posterior to the diaphragm.

Conclusion: We highlight the use of an US-guided and fluoroscopy-assisted posterior approach for use in celiac plexus neurolysis procedures, particularly in patients suffering from contraindications from the US or EUS-guided anterior approaches.

Keywords: ultrasound, image-guidance, posterior approach, celiac plexus neurolysis, pancreatic cancer, abdominal pain

\section{Background}

Severe abdominal pain is common in patients with pancreatic cancer and in most cases, requires the chronic use of high-dose narcotic analgesics. However, this form of treatment has several consequential adverse effects. ${ }^{1-4}$ An alternative method to effectively reduce intractable pain in abdominal malignancies is to block nociceptive impulses at the level of the celiac plexus. ${ }^{5}$

Image-guided celiac plexus block and neurolysis are important therapeutic options for the management of refractory abdominal pain. ${ }^{6,7}$ The celiac plexus, located in the retroperitoneal position of the upper abdomen at the level of the T12 and L1 vertebrae lie anterior to the crura of the diaphragm. Several posterior percutaneous approaches have been used to block the celiac plexus, including classic retrocrural, anterocrural, transaortic, and transdiscal approaches. Recent studies have highlighted the use of ultrasound for celiac plexus blockade and 
neurolysis using the transabdominal approach. ${ }^{8}$ In this study, we report a case in which an ultrasound-guided posterior retrocrural approach was employed for celiac plexus neurolysis with fluoroscopy-assisted needle positioning and extension of the injection.

\section{Case Report}

Informed consent for the publication of the case details and pictures was obtained from the patient and his family. The publication of this case and accompanying pictures has been approved by the ethics committee of Beijing Chaoyang Hospital, Capital Medical University. A 60year-old male with progressive jaundice and weight loss was diagnosed with pancreatic cancer. According to computed tomography (CT) assessments, an uncinate process occupied the head of the pancreas and distal common bile duct, leading to dilatation of the intrahepatic and extrahepatic bile ducts. Upon first admission to our hospital, the patient was treated with percutaneous transhepatic cholangial drainage (PTCD). His comorbidities included type 2 diabetes and coronary heart disease. The patient received a resection of T2N0M0 lung cancer two years earlier. This was his second admission to our hospital as a result of abdominal pain, nausea and vomiting for two weeks. He was diagnosed with biliary obstruction and underwent duodenal and biliary stent implantation which did not relieve his abdominal pain. Oxycodone was administered orally for pain control, followed by a fentanyl transdermal at a dose of $75 \mathrm{mcg}$ per hour. The patient also received two transfusions of $200 \mathrm{~mL}$ of red blood cells (RBC) suspension due to gastrointestinal hemorrhage.

As the persistent pain was difficult to relieve, $10 \mathrm{mg}$ of morphine was injected subcutaneously once every 4 hours. However, estimated pain relief based on the visual analogue scale (VAS) was low, with a score that persisted between 6 and 10, validated by continuous daily oral opioid consumption with an average morphine equivalent daily dose (MEDD) of up to $480 \mathrm{mg}$. The pain seriously impacted the patient's quality of life including diet, sleep pattern and mood. To alleviate the pain, celiac plexus neurolysis was proposed following consent from the patient and his family.

Intravenous access was established after arrival at the operating room. The patient's vitals including heart rate, arterial pressure, arterial oxygen saturation, and electrocardiography (ECG) were monitored throughout the operation. The prone position was adopted with a pillow set below the abdomen of the patient. Sufentanil $(5 \mu \mathrm{g})$ and oxygen $(5 \mathrm{~L} / \mathrm{min})$ were delivered by a facemask in response to complaints of intolerable pain. US scan was performed using a Clover US System (Huasheng, Inc., Shenzhen, Guangdong, China) with a curved array probe (C5-1, 5-2 MHz frequency). US gel was applied to the skin on the thoracolumbar region to achieve adequate acoustic coupling. The transducer was held in the nondominant hand of the operator, and placed below the costal margin in the short axis view (Figure 1). Upon identification of the three bony structures (the spinous process, articular process, and the vertebral body), the transducer was moved laterally and rotated anticlockwise until the diaphragm, erector spinae and psoas major muscles were identified (Figure 2). US images were optimized through the adjustment of depth, penetration frequency range, and gain. The orientation marker was directed laterally, and the position of the transducer was marked on the back of the patient using a skin-marking pen.

Following appropriate sterile techniques, the gel was used for the US transducer, after which the transducer and cable were blanketed with a sterile plastic sleeve. After that, local infiltration of $2 \%$ lidocaine at the puncture site, a puncture needle with an ultrasound enhanced tip was inserted in-plane aiming for the lateral anterior side of the vertebral body. The needle angle was adjusted for optimization whilst in the erector spinae muscle and then was slowly advanced into the target based on US imaging. Needle visibility was exemplified by tissue movement, a bright spot in consistence with the needle tip, or at the times the needle body on the US scanning. After that, the US probe was removed and the position of the needle tip was marked with $1 \mathrm{~mL}$ of contrast dye within both the anterior-posterior (AP) and lateral views (Figure 3) by the $\mathrm{C}$-arm. The correct needle tip position was confirmed by the $\mathrm{C}$-arm after extension of the contrast material located anterior to the vertebral body as well as posterior to the diaphragm.

Ropivacaine ( $5 \mathrm{~mL}, 0.5 \%$ in sodium chloride) was administered and pain relief was assessed based on the VAS scale ( 8 to 3 ) after $10 \mathrm{~min}$. The heart rate of the patient did not increase by $10 \%$ from baseline. SBP did not increase by more than $15 \mathrm{mmHg}$. Chemical neurolysis was performed with $8 \mathrm{~mL}$ of $98 \%$ alcohol injected into the left side. Prior to withdrawal of the needle, $3 \mathrm{~mL}$ of normal saline was injected to prevent a burning sensation that results from leakage of the neurolytic agents into the puncture route. All procedures were repeated on the other 

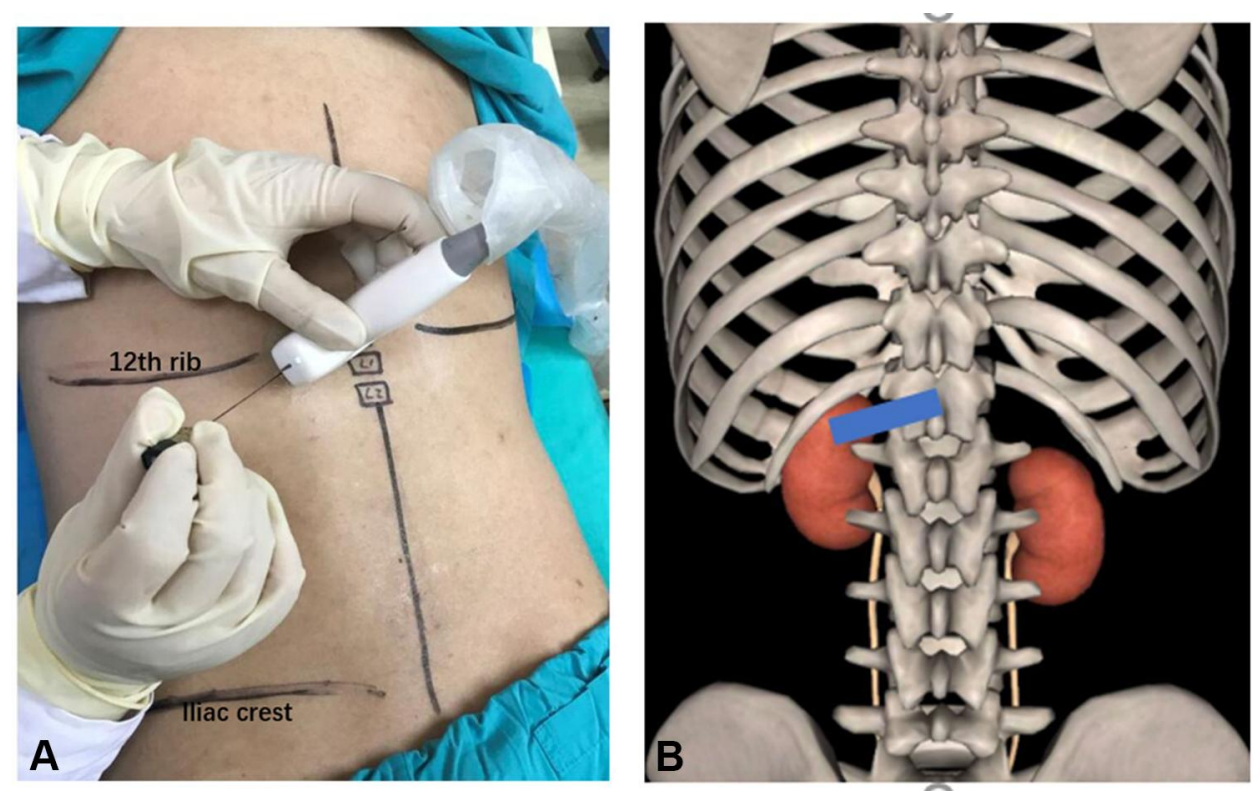

Figure I US-probe is placed below the costal margin in the short oblique axis-view for posterior celiac plexus neurolysis.

Notes: (A) The transducer is held in the non-dominant hand of the operator, and placed below the costal margin in the short oblique axis-view. (B) This transverse oblique scanning paralleling the 12 th rib provides a large acoustic window without obstacle of the rib, allowing the needle to reach the TI2 and LI vertebral bodies directly.

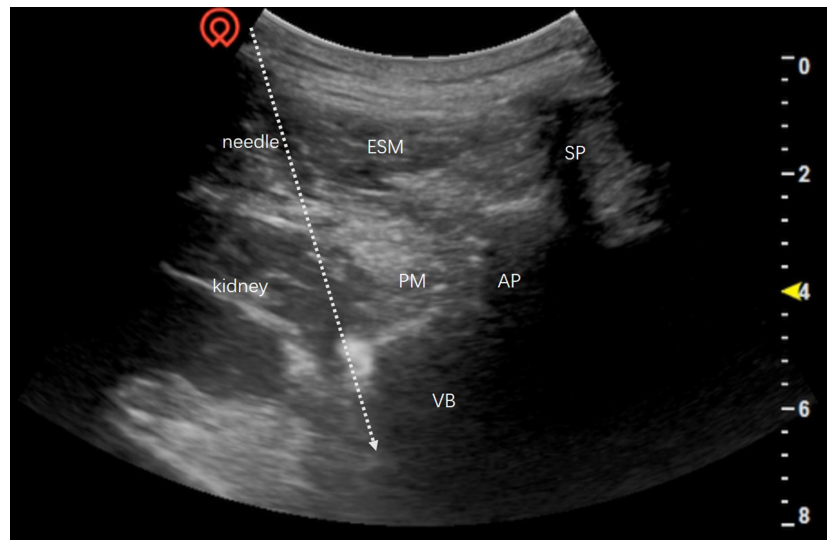

Figure 2 This sonogram shows the needle trajectory from the lateral inferior side to the anterior superior side, towards the lateral anterior side of the vertebral body. Notes: The US-guided in-plane puncture technique shows the needle trajectory and surrounding structures such as erector spinae muscle (ESM), psoas muscle (PM), spinous process (SP), articular process (AP) and vertebral body (VB). Abbreviations: ESM, erector spinae muscle; PM, psoas muscle; SP, spinous process; AP, articular process; VB, vertebral body.

side and the achieved effective pain relief, reflected by a VAS score of 3 and an average MEDD of $80 \mathrm{mg}$.

\section{Discussion}

Celiac plexus neurolysis (CPN) is an efficient method to interrupt the transmission of pain that originates at the celiac plexus. To date, multiple US-guided approaches have been implemented including EUS-guided and USguided anterior CPN. However, a US-guided posterior approach has not yet been reported. To our knowledge, the introduction of EUS-led CPN was made by Wiersema et al in 1996. The celiac ganglia typically appear as converged small spheres with hypoechoic bands. ${ }^{9,10}$ EUS-led CPN is simple and can be performed in 2 to 3 minutes. Real-time performance is dependent on the prerequisite of the Doppler control of the vessel interposition. However, either general anesthesia or deep intravenous sedation is required to perform this procedure. The US technique uses a transabdominal approach which allows CPN to be performed in patients that cannot bear prone or lateral decubitus positioning. ${ }^{11}$ Numerous studies have demonstrated the effectiveness of the US or EUS-guided anterior approach to alleviate pain. However, the patient had a history of gastrointestinal hemorrhage and biliary and duodenal stents for biliary obstruction, which are contraindicated for the US or the EUS-guided anterior approaches. So, US or EUS-guided anterior approaches are not recommended for running the risk of gastric perforation, retroperitoneal abscesses and the accidental destruction of stents.

Therefore, the posterior approach for CPN was offered for controlling intractable abdominal pain resulting from pancreatic cancer. Due to common posteriors, two-needle sets are used, one on each side at the level of the L1 vertebral body angling to the T12 vertebral body. ${ }^{11}$ For the retrocrural approach, fluoroscopy unfolds the extension 

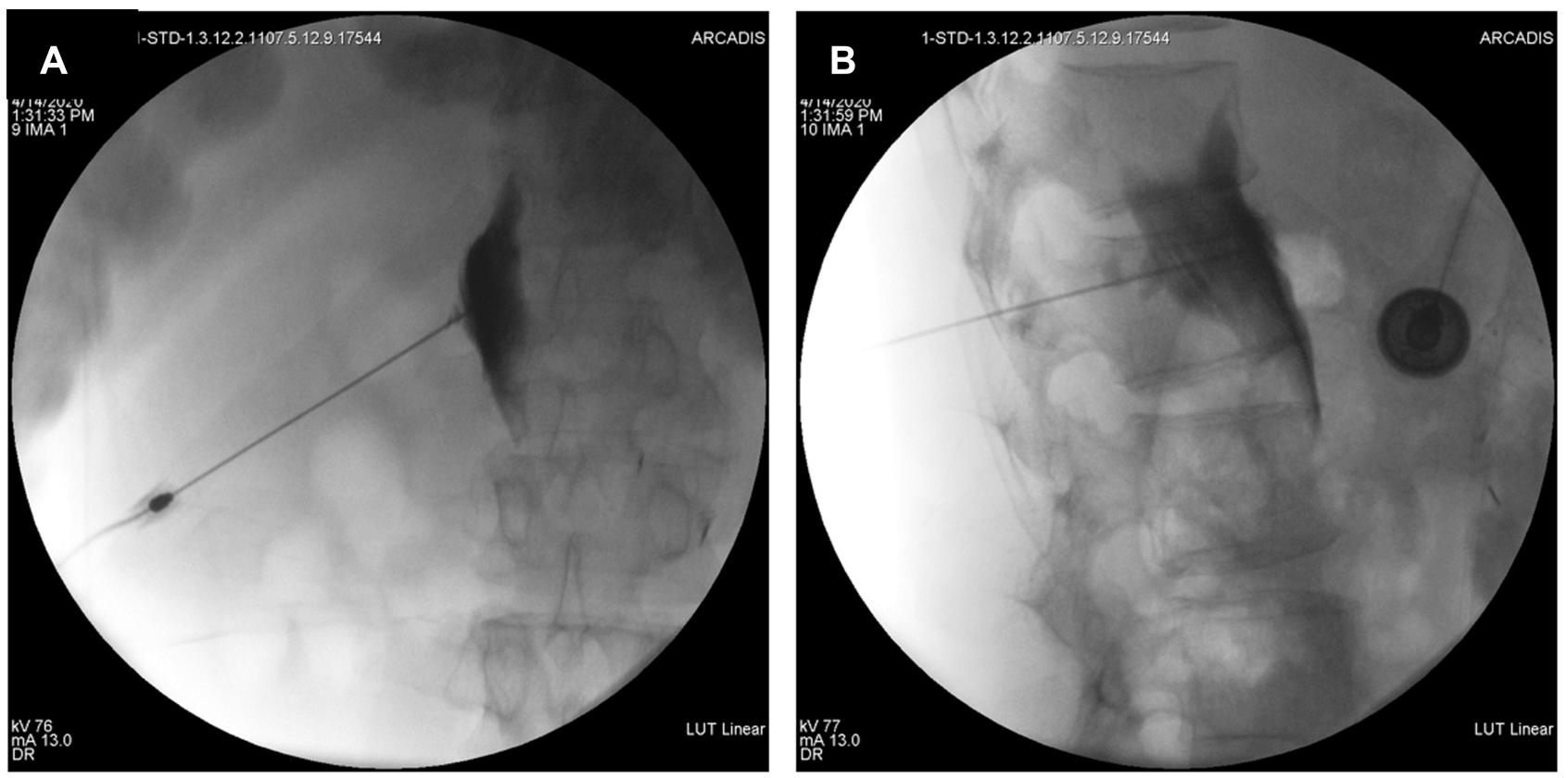

Figure 3 The position of the needle tip is marked with $1 \mathrm{~mL}$ of contrast dye within both the anterior-posterior (AP) and lateral views by the C-arm.

Notes: (A) Anterior-posterior image shows the spread of contrast anterior to the vertebral bodies. (B) Lateral image shows the spread of the contrast anterior to the vertebral bodies and posterior to the diaphragm.

Abbreviation: AP, anterior-posterior.

of the contrast material anterior to the vertebral body and posterior to the diaphragm. For the anterocrural approach, the needle tip is advanced through the diaphragm, which is more challenging on the left as a result of the location of the aorta. Regarding the transaortic approach, the needle can be slowly advanced through the aorta to the celiac plexus. For the transdiscal method for the celiac plexus, the needle is set using the T12-L1 intervertebral disc, which cannot be routinely applied in clinical practice because of the high risks of diskitis, disk herniation, and spinal cord puncture. ${ }^{12-14}$ Because the patient had anemia due to gastrointestinal hemorrhage, we provided the retrocrural approach to avoid intravascular injection and bleeding.

Imaging guidance for posterior approach CPN has evolved due to advances in imaging processes including fluoroscopy, CT and magnetic resonance (MR). ${ }^{14}$ Although popular and simple to perform, fluoroscopy provides relatively poor anatomical resolution, making it difficult to distinguish the celiac plexus from adjacent structures such as the pancreas, blood vessels, tumors, and lymph nodes. Consequently, fluoroscopy-guided celiac plexus neurolysis is associated with higher complication rates and has been surpassed by CT. ${ }^{4,15-17}$ Coupled with retroperitoneal structures, CT can detect tumor expansion in addition to other causes of abdominal pain, including duodenal obstructions, bone destruction and muscle invasion. ${ }^{14-16}$ In addition, MRI provides superior soft-tissue resolution without exposure to ionizing radiation. However, MRI is more expensive and requires more complex imaging equipment. ${ }^{15}$

In our hospital, a large number of patients are waiting for CT examination and CT cannot be routinely used for interventional pain therapies. So, we used US-guided and fluoroscopy-assisted posterior approach CPN for the treatment of abdominal pain in a patient with pancreatic cancer. This case confirmed the unique advantages of US guidance during interventional pain procedures. Firstly, US guidance has the advantage of unnecessary radiation exposure for the operators, not merely for patients with short life expectancy. Secondly, US machines are much more portable and cheaper compared to $\mathrm{CT}$ and MR devices. Thirdly, we used US guidance to visualize soft tissues, vessels and the kidneys to prevent renal injuries, avoiding the disadvantages of $\mathrm{x}$-rays as a single imaging modality. The duration of the procedure was also reduced using our method. Finally, the most important advantage was transverse oblique scanning paralleling the 12 th rib. This provided a large acoustic window without obstacle of the rib, allowing the needle to directly reach the T12 and L1 vertebral bodies. According to the distribution of $1 \mathrm{~mL}$ of the contrast dye, the needle was adjusted, and the tip affirmed with the assistance of fluoroscopy. 
Actually, intrathecal drug delivery ought to be another choice for cancer patients, however the patient was diagnosed with pancreatic cancer osseous metastasis and refused this treatment. US-guided and fluoroscopy-assisted posterior CPN was successfully achieved in this case. On the one hand, the US-guided posterior approach to CPN operation obtained successful real-time visibility of the vessels and kidney. On the other hand, the diffusion of the neurolytic agent was visible and confirmed through the aid of the C-arm. Despite these advances, the technology had some limitations. Firstly, the aorta and visceral arteries were not completely visualized during the US-guided posterior approach, thus bleeding cannot be completely avoided. Secondly, the technique requires a highly skilled and experienced operator along with the cooperation of the patient in the prone position.

Finally, this technique is indicated for pain associated with pancreatic cancer, especially beneficial for patients who have intolerable adverse effects of opioid therapy such as drowsiness, somnolence, confusion, delirium, dry mouth, anorexia, constipation, nausea, and vomiting, or have contraindications for EUS-guided and US-guided anterior CPN.

\section{Conclusions}

This US-guided and fluoroscopy-assisted posterior approach in CPN can provide a new option for patients with intractable abdominal pain as a key component of multidisciplinary management.

\section{Funding}

This project was supported by grants NO. Z181100001718222 from the clinical research project of the Beijing Municipal Science \& Technology Commission (to Dr. Ma).

\section{Disclosure}

The authors declare no conflicts of interest for this work.

\section{References}

1. Eisenberg E, Carr DB, Chalmers TC. Neurolytic celiac plexus block for treatment of cancer pain: a meta-analysis. Anesth Analg. 1995;80 (2):290-295. doi:10.1097/00000539-199502000-00015
2. Kaufman M, Singh G, Das S, et al. Efficacy of endoscopic ultrasound-guided celiac plexus block and celiac plexus neurolysis for managing abdominal pain associated with chronic pancreatitis and pancreatic cancer. J Clin Gastroenterol. 2010;44(2):127-134. doi:10.1097/MCG.0b013e3181bb854d

3. Kelsen DP, Portenoy R, Thaler H, Tao Y, Brennan M. Pain as a predictor of outcome in patients with operable pancreatic carcinoma.. Surgery. 1997;122(1):53-59. doi:10.1016/S0039-6060(97)90264-6

4. Markman JD, Philip A. Interventional approaches to pain management. Med Clin North Am. 2007;91(2):271-286. doi:10.1016/j.mcna.2006. 10.015

5. De Cicco M, Matovic M, Balestreri L, Fracasso A, Morassut S, Testa V. Single-needle celiac plexus block: is needle tip position critical in patients with no regional anatomic distortions? Anesthesiology. 1997;87(6):1301-1308. doi:10.1097/00000542-199712000-00007

6. Fujita Y, Sari A. Max Kappis and the celiac plexus block. Anesthesiology. 1997;86(2):508. doi:10.1097/00000542-199702000-00033

7. Penman ID. Coeliac plexus neurolysis. Best Pract Res Clin Gastroenterol. 2009;23(5):761-766. doi:10.1016/j.bpg.2009.05.003

8. Giménez A, Martínez-Noguera A, Donoso L, Catalá E, Serra R. Percutaneous neurolysis of the celiac plexus via the anterior approach with sonographic guidance. Am J Roentgenol. 1993;161(5):10611063. doi:10.2214/ajr.161.5.8273610

9. Sakamoto H, Kitano M, Komaki T, Imai H, Kamata K, Kudo M. Endoscopic ultrasound-guided neurolysis in pancreatic cancer. Pancreatology. 2011;11(2):52-58. doi:10.1159/000323513

10. Wiersema MJ, Wiersema LM. Endosonography-guided celiac plexus neurolysis. Gastrointest Endosc. 1996;44(6):656-662. doi:10.1016/ S0016-5107(96)70047-0

11. Bahn BM, Erdek MA. Celiac plexus block and neurolysis for pancreatic cancer. Curr Pain Headache Rep. 2013;17(2). doi:10.1007/ s11916-012-0310-y

12. Ina H, Kitoh $\mathrm{T}$, Kobayashi $\mathrm{M}$, Imai S, Ofusa $\mathrm{Y}$, Goto H. New technique for the neurolytic celiac plexus block: the transintervertebral disc approach. Anesthesiology. 1996;85(1):212-217. doi:10.1097/ 00000542-199607000-00028

13. Titton RL, Lucey BC, Gervais DA, Boland GW, Mueller PR. Celiac plexus block: a palliative tool underused by radiologists. Am J Roentgenol. 2002;179(3):633-636. doi:10.2214/ajr.179.3.1790633

14. Wang PJ, Shang M, Qian Z, Shao CW, Wang JH, Zhao XH. CTguided percutaneous neurolytic celiac plexus block technique. Abdom Imaging. 2006;31(6):710-718. doi:10.1007/s00261-006-9153-5

15. Kambadakone A, Thabet A, Gervais DA, Mueller PR, Arellano RS. CT-guided celiac plexus neurolysis: a review of anatomy, indications, technique, and tips for successful treatment. RadioGraphics. 2011;31 (6):1599-1621. doi:10.1148/rg.316115526

16. Loukas M, Klaassen Z, Merbs W, Tubbs RS, Gielecki J, Zurada A. A review of the thoracic splanchnic nerves and celiac ganglia. Clin Anat. 2010;23(5):512-522. doi:10.1002/ca.20964

17. Sekhadia M, Chekka K. Standards of care for celiac plexus neurolysis: radiologic guidance and fellowship training. J Support Oncol. 2009;7(3):89-90. doi:http://dx.doi.org/ 


\section{Publish your work in this journal}

The Journal of Pain Research is an international, peer reviewed, open access, online journal that welcomes laboratory and clinical findings in the fields of pain research and the prevention and management of pain. Original research, reviews, symposium reports, hypothesis formation and commentaries are all considered for publication. The manuscript management system is completely online and includes a very quick and fair peer-review system, which is all easy to use. Visit http:// www.dovepress.com/testimonials.php to read real quotes from published authors. 